\title{
Natalizumab improves neurological function in MS
}

New data suggest that natalizumab can improve neurological functioning as well as slowing the progression of disability in patients with multiple sclerosis (MS). In this post-hoc analysis of data from the AFFIRM trial-a randomized, doubleblinded, placebo-controlled study of patients with relapsing MS-Theodore Phillips and colleagues identified and defined a sustained neurological improvement in natalizumab-treated patients, as measured by the EDSS (Kurtzke Expanded Disability Status Scale). "The sustained EDSS improvements correlate to changes in blinded, patient-reported changes in overall well-being," explains Phillips. "We observed a significant and clinically meaningful natalizumab treatment effect versus placebo."

The researchers analyzed data from 620 patients, who were treated with placebo or $300 \mathrm{mg}$ of natalizumab (a monoclonal antibody against the cell adhesion molecule $\alpha 4$-integrin) for up to 2 years, and who had a baseline EDSS score $\geq 2.0$. The cumulative probability of improvement (defined as $\mathrm{a} \geq 1.0$ point

\author{
YThe ... probability of \\ improvement ... was increased \\ by $69 \%$ in natalizumab-treated \\ patients with relapsing MS 77
}

decrease in EDSS score sustained for $\geq 12$ weeks) was increased by $69 \%$ in natalizumab-treated patients with relapsing MS, which also correlated with improved quality of life. Importantly, use of more-stringent criteria for improvement (1.5 point and 2.0 point decreases in EDSS scores sustained for 36 weeks and 48 weeks, respectively) also revealed evidence of a significant benefit of natalizumab treatment.

Intriguingly, during the first year of the study, the beneficial effect of natalizumab treatment was greater in patients who relapsed than in those who did not, whereas in the second year the effect was greater in patients who did not relapse. "These findings suggest as yet undiscovered, natalizumab-induced pro-repair effects involving immune cells or CNS mechanisms," comments Phillips. "A better understanding of natalizumab's indirect or possibly direct effects on molecular mechanisms involved in improvement of CNS function in MS should lead down some interesting paths."

Phillips and colleagues are now evaluating the neurological improvements associated with natalizumab treatment by means of another standardized MS clinical outcome measure, the Multiple Sclerosis Functional Composite score. "Such familiar methodologies used to demonstrate a robust, clinically meaningful and sustained EDSS improvement should be further validated, and should be easily applicable to future prospective clinical treatment trials in MS," concludes Phillips.

Iley Ozerlat

Original article Phillips, J. T. et al. Sustained improvement in expanded disability status scale as a new efficacy measure of neurological change in multiple sclerosis: treatment effects with natalizumab in patients with relapsing multiple sclerosis. Mult. Scler. doi:10.1177/1352458511399611 